\title{
Physiological and molecular analysis of OSTPS30 by gamma irradiation
}

\author{
Se Won Kim • In Jung Jung • Sang Hoon Kim $\cdot$ Hong-Il Choi $\cdot$ Si-Yong Kang • Jin-Baek Kim
}

Received: 1 February 2019 / Revised: 29 March 2019 / Accepted: 29 March 2019

(C) Korean Society for Plant Biotechnology

\begin{abstract}
Terpenes constitute a large class of secondary metabolites in plants. The Oryza sativa terpene synthase is a vital gene in plant defense response. In this study, the molecular and physiological functions of Oryza sativa terpene synthase 30 (OsTPS30, LOC_Os08g07080) were investigated after exposure of the seeds and plants to gamma-rays. The OSTPS30 expression was slightly induced at 200 Gray (Gy), but was significantly induced at $400 \mathrm{~Gy}$. The total terpenoid was synthesized more in OsTPS30overexpressing (OX-OsTPS30) Arabidopsis thaliana plants than in wild-type (WT) plants. The OX-OsTPS30 plants exhibited resistance to gamma-rays, as compared to WT. The OX-OsTPS30 plants had significantly increased height and weight after gamma irradiation. Additionally, the activity of antioxidant enzymes was increased more in OX OsTPS30 plants than in WT plants after gamma irradiation. Furthermore, the OsTPS30-GFP fusion protein was mostly localized in the chloroplast, suggesting that OsTPS30 is putative MEP pathway-related terpene synthase.
\end{abstract}

Keywords Terpene synthase, gamma-ray, chloroplast, MEP pathway

\section{Introduction}

Terpenes, which constitute a large class of secondary metabolites in plants, have been divided into the following classes: hemiterpenes (C5); monoterpenes (C10); sesquiterpenes (C15); diterpenes (C20); sesterterpenes (C25); triterpenes (C30); tetraterpenes (C40) and polyterpenes (> C40) (Tholl 2006; Singh and Sharma 2015). Most plant genomes include a medium-sized family of genes encoding terpene synthases

S. W. Kim - I. J. Jung • S. H. Kim · H.-I. Choi · S.-Y. Kang J.-B. $\operatorname{Kim}(\varangle)$

Advanced Radiation Technology Institute, Korea Atomic Energy Research Institute (KAERI), Jeongeup, Jeollabuk 56212, Korea e-mail: jbkim74@kaeri.re.kr
(TPSs) (Chen et al. 2011). There are two terpene biosynthesis pathways in plants, namely the chloroplast 2-C-methylerythritol-4-phosphate (MEP) pathway and cytosolic mevalonate (MEV) pathways, both of which require a 5-carbon isoprenoid diphosphate precursor for the production of all terpenes. Based on their phylogenetic relationships, the plant TPS genes are distributed in seven different clades. The angiosperm clades consist of TPS-a, TPS-b, and TPS-g. The TPS-a clade comprises the sesquiterpene synthases, while the TPS-b and TPS-g clades consist mainly of monoterpene synthases and sesquiterpene synthases, respectively. The TPS-d clade includes monoterpene, sesquiterpene, and diterpene synthases. Meanwhile, the TPS-h clade consists of diterpene synthases (Bohlmann et al. 1998; Dudareva et al. 2006; Chen et al. 2011; Li et al. 2012).

The most studied TPS gene family has been that of Arabidopsis thaliana (Sun et al. 1994; Yamaguchi et al. 1998; Bohlmann et al. 2000; Chen et al. 2003; Fäldt et al. 2003; Chen et al. 2004; Tholl et al. 2005; Herde et al. 2008), with only a few TPSs having been isolated in cultivated plants, including rice (Oryza sativa), tomato (Solanum lycopersicum), grape (Vitis vinifera), poplar (Populus trichocarpa), and sorghum (Sorghum bicolor) (Falara et al. 2011; Irmisch et al. 2014; Lee et al. 2015; Yoshitomi et al. 2016; Zhang et al. 2016). However, a detailed analysis of the presence and role of terpenes in these plants has not been published. Monoterpene synthases generally comprise 600-650 amino acid residues, while sesquiterpene synthase have 50-70 fewer amino acids (Martin et al. 2004). All TPS C-terminals contain an aspartate-rich DDxxD domain, which helps coordinate divalent metal ions for a subsequent binding of a substrate (Lesburg et al. 1997; Peterd et al. 2003; Tholl 2006; Tholl et al. 2011). All plant terpenoids are biosynthesized by TPSs, with monoterpene and diterpene synthases localized to the chloroplast via the MEP pathway, and the sesquiterpene synthases present in the cytoplasm via MEV pathway (Aharoni et al. 2003; Singh and Sharma 2015; Chen et al. 2011; 
Tholl et al. 2011). Tomato TPS are localized in mitochondria, but the in vivo substrates are currently unknown (Falara et al. 2011).

Some plant TPSs effect primary metabolism [e.g., gibberellin biosynthesis], but most TPSs are involved in the biosynthesis of secondary metabolites in response to environmental stimuli (Chen et al. 2011; Cheng et al. 2007). There has been recent progress in the characterization of the biochemistry and molecular genetics underlying terpenoid biosynthesis. Additionally, the biological functions of many terpene compounds associated with phytohormone biosynthesis have been determined. For example, jasmonate-responsive volatile terpenoids are important for defense responses in rice and help protect plant from UV radiation and photooxidative stress (Jenkins et al. 2009; Yohitomi et al. 2016). Moreover, terpenes provide protection from heat stress and also contribute to membrane stabilization, resistance to insects and microorganisms, and plant-plant signaling (Loreto et al. 1998; Copolovici et al. 2005; Baldwin et al. 2006; Keeling et al. 2006).

The rice genome has 33 TPS genes. OsTPS18 has localized in the cytoplasm and synthesized sesquiterpenes $(E)$ nerolidol and (E)- $\beta$-farnesene (Kiryu et al. 2018). OsTPS19 acts as an $(S)$-lemonine synthetase in rice and defends against Magnaporthe oryzae by inhibiting spore germination (Chen et al. 2018). OsTPS20 plays a major role in producing terpene volatiles during abiotic stress (Lee et al. 2015). OsTPS46 plays an important role in rice defense against Rhopalosiphum padi. (Sun et al. 2017). Additionally, a previous study revealed that the gamma irradiation of rice seedlings upregulated the expression of OSTPS19, OsTPS20, OsTPS25, OsTPS26, and OsTPS30, although the effect on OSTPS30 was inconclusive (Lee et al. 2015). In this study, we analyzed molecular and physiological function of OsTPS30 by gamma irradiation.

\section{Materials and methods}

Plant materials and gamma irradiation

Rice plants (Oryza sativa spp. japonica cv. Ilpoom) were grown in half-strength Murashige and Skoog (MS) agar medium (Duchefa Biochemie, Haarlem, Netherlands) and cultured aseptically in a growth chamber at $28^{\circ} \mathrm{C}$ under a 16-h light/8-h dark cycle. The WT and OX OsTPS30 plants in $A$. thaliana were sown in plates containing half-strength MS agar medium with $1.5 \%$ of sucrose (Sigma-Aldrich, St. Louis, USA). The plates were incubated for 3 days at $4{ }^{\circ} \mathrm{C}$ in darkness, and then transferred to a growth chamber for a subsequent incubation at $22^{\circ} \mathrm{C}$ with a $16-\mathrm{h}$ light/8-h dark cycle and $70 \%$ of humidity. For the gamma-ray treatment, rice seeds were exposed to 100,200 , or 400 Gy for $24 \mathrm{~h}$ using a ${ }^{60} \mathrm{Co}$ gamma irradiator $(150 \mathrm{TBq}$ capacity; Atomic Energy of Canada Limited, Ottawa, Canada) at the Korea Atomic Energy Research Institute. A. thaliana plants during the vegetative stage and plants with fully expanded rosette leaves were independently irradiated 18 days after seeding. Plants were exposed to gamma-rays at 100, 200, 300, 400, or $500 \mathrm{~Gy}$ for $24 \mathrm{~h}$, and the resulting phenotypes were evaluated after 14 days.

RNA isolation and quantitative real time polymerase chain reaction (qRT-PCR)

Total RNA was extracted from the leaves using the RNeasy Plant Mini Kit (Qiagen, Hilden, Germany), after which the concentration and quality were assessed with a Nanodrop 2000 spectrophotometer (Thermo Fisher Scientific, Waltham, USA). The RNA was then used as the template to synthesize first-strand cDNA using the ReverTra Ace- $\alpha$ - kit (Toyobo, Osaka, Japan). The qRT-PCR was conducted using the iQ тм SYBR ${ }^{\circledR}$ Green Supermix (Bio-Rad, Hercules, USA), and the CFX96 Touch Real-Time PCR Detection System (BioRad, Hercules, USA). Details regarding the qRT-PCR primers are listed in Table 1.

Table 1 Primers used for the qRT-PCR analysis

\begin{tabular}{cll}
\hline Gene & \multicolumn{1}{c}{ Primer name } & \multicolumn{1}{c}{ Sequence $\left(5^{`}\right.$ to $\left.3^{`}\right)$} \\
\hline OsTPS30 & OsTPS30-F & ATGAAAGAACGGGTTCAAGTTGTGA \\
& OsTPS30-R & AATGTTATACGGCTCCATATAGAGCGAT \\
& OsTPS30-qRT-F & ACCACCACCATGCTTCTACA \\
OsNAC10 & OsTPS30-qRT-R & ACTCCTTTATCCCAACGCAT \\
& OsNAC10-qRT-F & TACACAACACCTCATCCAA \\
OsActin & OsNAC10-qRT-R & ATGATCTAGGCGTGACTC \\
& OsACT-qRT-R & TGAAGTGCGACGTGGATATTAG \\
& AtACT2-qRT-F & CAGTGATCTCCTTGCTCATCC \\
& AtACT2-qRT-R & GCCATCCAAGCTGTTCTCTC \\
\end{tabular}


Cloning of OsTPS30 and the production of transgenic plants

The OsTPS30 gene was amplified by PCR using genespecific primers. The PCR product was inserted into the $\mathrm{pCR}^{\mathrm{TM}} 8 / \mathrm{GW} / \mathrm{TOPO}^{\circledR}$ vector (Invitrogen, Carlsbad, USA) and sequenced. After the sequence was verified, it was integrated into the pMDC83 destination vector using the recombination-based Gateway Cloning System (Invitrogen, Carlsbad, USA). The CaMV35S:OsTPS30-green fluorescence protein (GFP) construct was inserted into A. thaliana cells using an Agrobacterium tumefaciens-mediated (strain GV3101) floral dip method (Clough et al. 1998).

Total terpenoids of transgenic plants

The harvested plant material was immediately frozen in liquid nitrogen. To homogenize the tissue, one glass bead and cold $95 \%$ methanol was added to each sample tube and homogenized with QIAGEN. Remove glass beads and incubate the sample at room temperature for $48 \mathrm{~h}$ in dark. Samples were centrifuged to collect the supernatant. 1.5 $\mathrm{mL}$ chloroform was added to each and $200 \mu \mathrm{l}$ sample supernatant was added to each. The Remark standard curve was prepared by diluting $200 \mu \mathrm{l}$ of Linalool (Sigma-Aldrich, St. Louis, USA) solution in $1.5 \mathrm{ml}$ of chloroform. The sample was vortexed and $100 \mu \mathrm{l}$ of sulfuric acid (Sigma-Aldrich, St. Louis, USA) was added. The tubes were incubated in the dark for $2 \mathrm{~h}$ at room temperature for analysis. The supernatant was discarded and the precipitate was completely dissolved in 95\% methanol. The sample was transferred to a colorimetric cuvette and absorbance was measured at $538 \mathrm{~nm}$ on EVOLUTION 260 BIO (Thermo Fisher Scientific, Waltham, USA) (Ghorai et al. 2012).

Measurement of antioxidant enzymes activity

For all antioxidant enzyme assays, the proteins from 18day-old leaves samples $(0.1 \mathrm{~g})$ were homogenized in 200 $\mathrm{mM}$ potassium phosphate buffer $(\mathrm{pH} 7.0)$ containing 0.1 $\mathrm{mM}$ ethylenediaminetetraacetic acid (EDTA) at $4^{\circ} \mathrm{C}$. After homogenization, the samples were centrifuged at 15,000 rpm for $15 \mathrm{~min}$ at $4^{\circ} \mathrm{C}$. Total protein content was determined by the Bradford assay using BSA as a standard (Bradford 1976). Peroxidase (POD) activity was determined with a modified Pütter (Pütter et al. 1974) method and Ascorbate peroxidase (APX) activity was analyzed according to the method of Mittler and Zilinskas (1993). Superoxide dismutase (SOD) activity was assayed using the sigma SOD determination kit (\#19160) (Sigma-Aldrich, St. Louis,
USA) following the manufacturer's instructions. Catalase (CAT) activity was assayed using the Amplex ${ }^{\circledR}$ Red Catalase Assay kit (A22180) (Thermo Fisher Scientific, Waltham, USA) following the manufacturer's instructions.

\section{Subcellular localization of OsTPS30}

A. thaliana protoplasts were isolated from the leaves of 14-days-old WT and OX-OsTPS30 plants (CaMV35S: OsTPS30GFP). All plants were incubated at $22{ }^{\circ} \mathrm{C}$ for 2 weeks under a 16-h light/8-h dark cycle. The fluorescence of the GFP and red chlorophyll autofluorescence was detected using the LSM800 confocal laser scanning microscope (Carl Zeiss, Jena, Germany) at an excitation wavelength of $488 \mathrm{~nm}$.

\section{Results}

OsTPS30 expression following gamma irradiation

To investigate the effect of gamma-ray on the expression of OsTPS30 expression, rice seeds were exposed to gamma-ray. A subsequent qRT-PCR revealed that OsTPS30 expression levels were upregulated in response to gamma-ray. Increasing the gamma-ray dosage from 0 to 400 Gy resulted in a 3.4-fold increase in the OSTPS30 transcript abundance (Fig. 1A). These observations were consistent with previously reported RT-PCR results (Lee et al. 2015). Moreover, the OsNAC10 expression was increased with increasing intensity of gamma-rays (Fig. 1B), which is reportedly upregulated by gamma irradiation (Park et al. 2014), was used as a positive control.

\section{Overexpression of OsTPS30 in A. thaliana}

The gamma-ray treatment clearly upregulated OSTPS30 expression. To assess the physiological function of OsTPS30 in plants irradiated with gamma-rays, we constructed OXOsTPS30 plants. The OsTPS30 gene was PCR-amplified from rice. The pMDC83-OsTPS30:GFP vector was inserted into A. tumefaciens GV3101 cells for subsequent transformation of $A$. thaliana using a floral dip method (Fig. 1C). The $T_{3}$ homozygous transgenic plant lines, which were resistant to hygromycin, were produced by the selfpollination of $\mathrm{T}_{2}$ transgenic plant lines. The OsTPS30 expression levels were evaluated by qRT-PCR (Fig. 1D). Three homozygous transgenic plant lines (\#2-1, \#4-6, \#9-17) with different expression levels were selected to analyze the OsTPS30 expression levels and the effects of the gamma-ray 
A

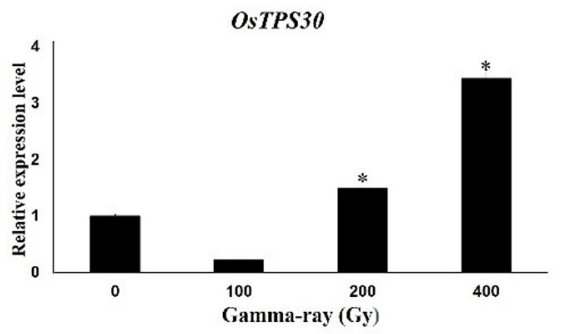

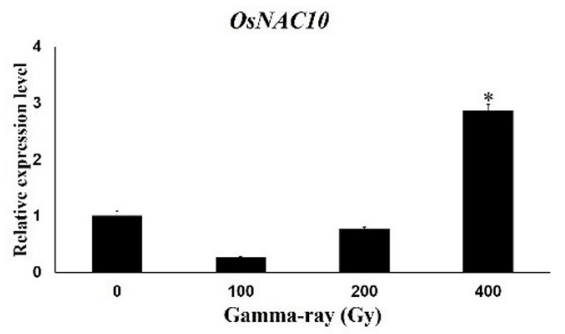

C

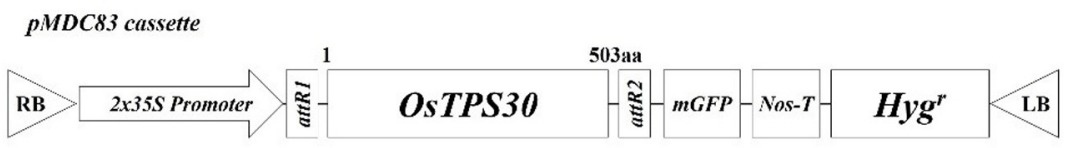

D

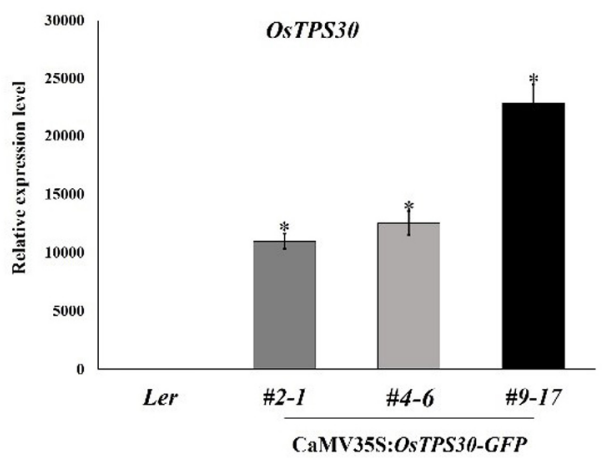

$\mathbf{E}$

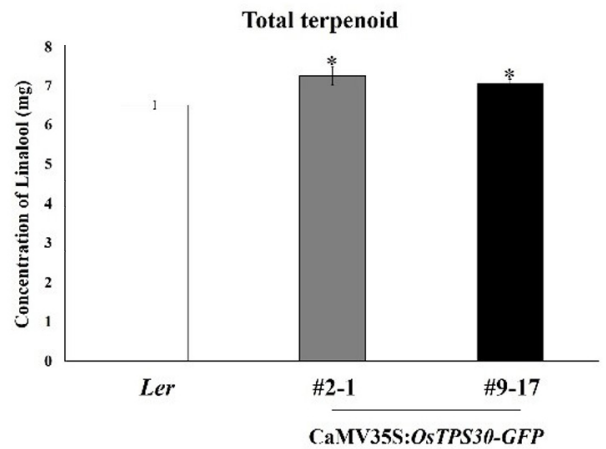

Fig. 1 Expression of OSTPS30 in rice plants exposed to gamma irradiation and overexpression of OsTPS30 in transgenic A. thaliana plants. (A) Rice seeds irradiated with gamma-rays at 100, 200, or $400 \mathrm{~Gy}$. (B) OSNAC10 was used as a positive control for the gamma irradiation. The OsTPS30 transcript levels were analyzed using qRT-PCR, and normalized against those of $O S A C T$. (C) Schematic diagram of the CaMV35S:OSTPS30-GFP plasmid construct used to generate the OsTPS30-overexpressing transgenic plant lines. (D) OsTPS30 transcript levels were analyzed using qRT-PCR. Transcript levels were standardized against those of AtACT2. (E) A linalool Analysis of total terpenoid of OX-OsTPS30 (\#2-1, \#9-17) and WT plants. Data is presented as the mean \pm standard deviation for the three biological replicates. Asterisks indicate significant differences in the data for non-irradiated plants (0 Gy) or for the WT plants (Ler) at $P<0.05$ (One-way ANOVA)

treatment.

Identification of OsTPS30 as terpene synthase

Linalool, a monoterpene, was used to compare the synthesis of total terpenoid from the OX-OsTPS30 plants (\#2-1, \# 9-17) and WT plants. As a result, OX-OSTPS30 plants synthesized more total terpenoid than WT plants (Fig. 1E).

\section{Effect of gamma irradiation on $O X$-OsTPS30 plants}

To confirm rice OsTPS30 can increase the tolerance of other plants to gamma irradiation, we generated transgenic A. thaliana lines producing the GFP-tagged OsTPS30 protein. The 18-day-old plants exposed to gamma-rays at doses of 100-500 Gy for $24 \mathrm{~h}$ were transferred to normal growth conditions for a recovery period. After 14 days, the results showed that the height and weight of OX-
OsTPS30 plants (\#2-1, \#4-6, \#9-17) were significantly higher than those of WT plants. The phenotypes of the OX-OSTPS30 and WT plants were not significantly different under normal growing conditions (Fig. 2). These results indicate that OsTPS30 contributes to gamma irradiation resistance.

Antioxidant enzyme activity of $O X$-OsTPS30 plant

Reactive oxygen species (ROS) scavenging is a common protective response to gamma irradiation. Activity of antioxidant enzymes, POD, APX, CAT and SOD, are induced by gamma irradiation (Moussa 2008). In order to determine the activities of the antioxidant enzyme of different dosages of gamma irradiation on OX- OsTPS30 (\#2-1, \#9-17) and WT plants, we measured the enzymatic activity of POD, CAT, APX and SOD (Fig. 3). At 200 Gy, the POD activity showed OX-OsTPS30 plants (\#2-1, \#9-17) were 
A
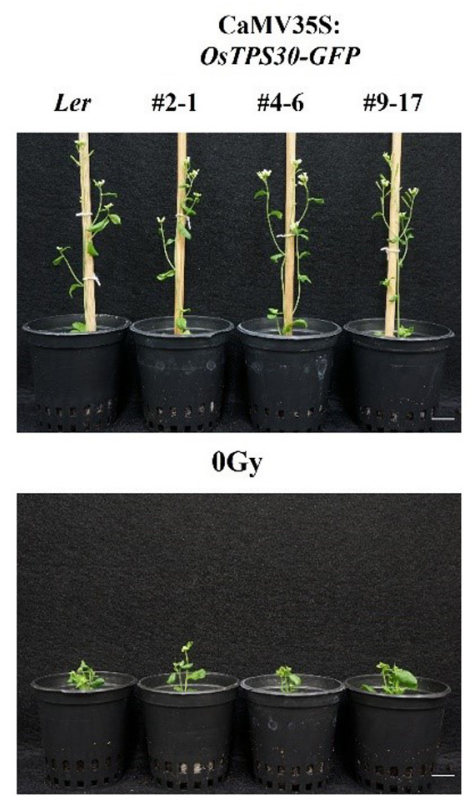

300Gy

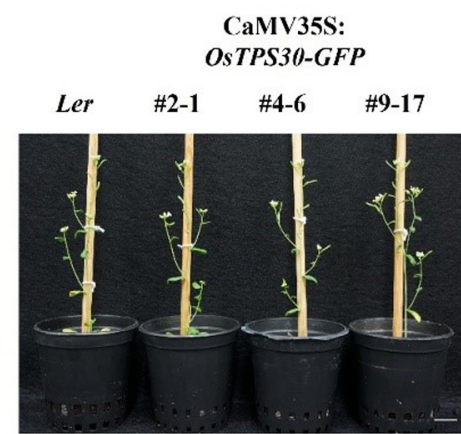

100Gy

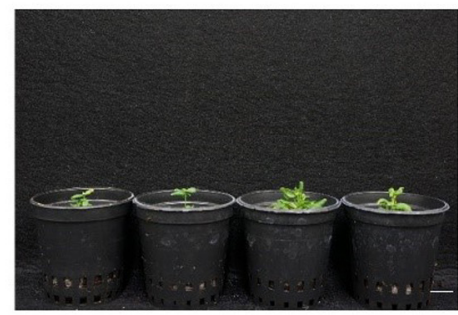

400Gy
CaMV35s:

OsTPS30-GFP

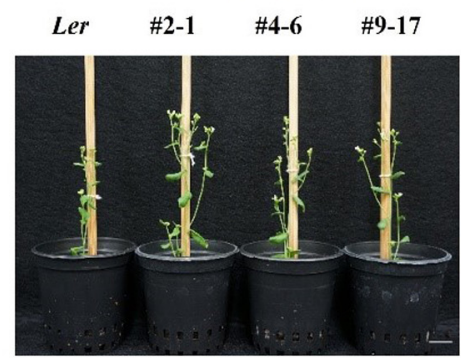

200Gy

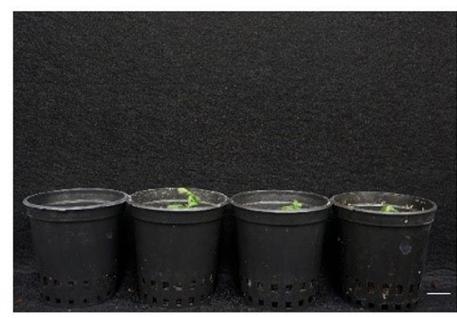

$500 G y$
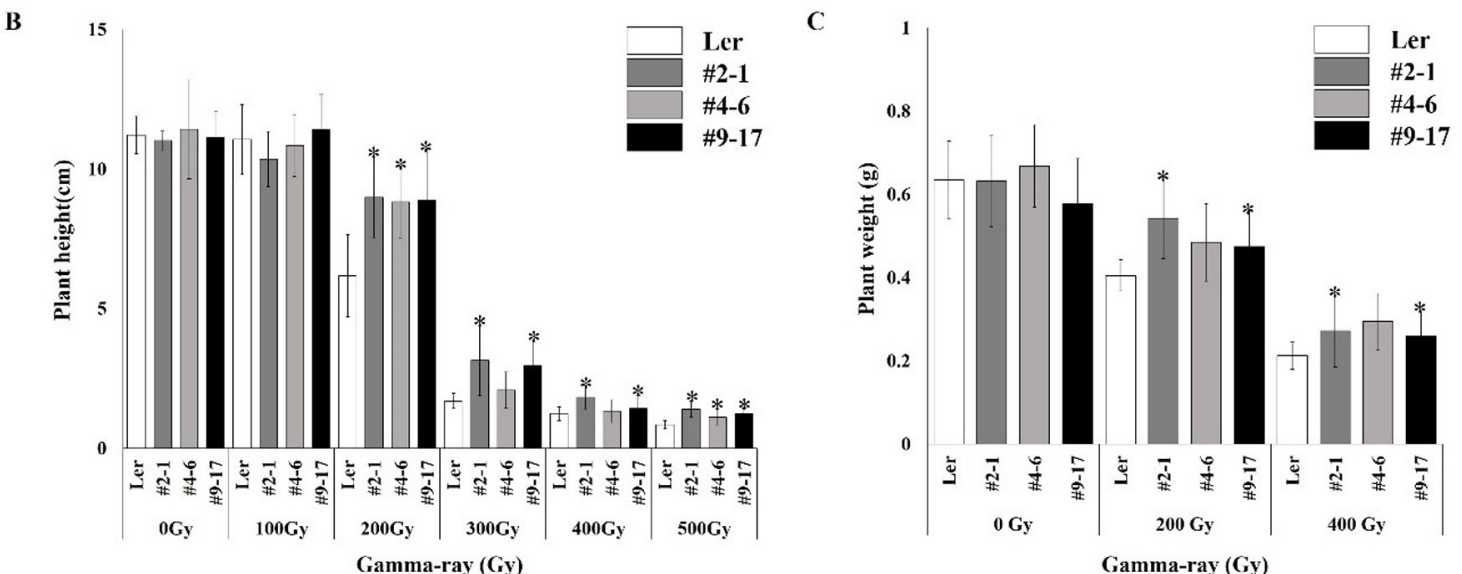

Gamma-ray (Gy)

Fig. 2 The OX-OsTPS30 plants enhances tolerance to gamma irradiation. (A) The OX-OsTPS30 and WT plants were grown for 18 days after seeding and then irradiated for $24 \mathrm{~h}$ at $0,100,200,300,400$, and $500 \mathrm{~Gy}$. The irradiated plants were then analyzed after 2 weeks. White bar $=1 \mathrm{~cm}$. (B) The height of OX-OsTPS30 and WT plants were also evaluated 2 weeks after the gamma irradiation. (C) The weight of OX-OsTPS30 and WT plants were evaluated 2 weeks after the gamma irradiation. The data is presented as the mean \pm standard deviation for the three biological replicates. Asterisks indicate significant differences in the data for the WT plants (Ler) at $P<0.05$ (One-way ANOVA)

significantly higher than those of WT plants. However, when OX-OsTPS30 plants (\#2-1, \#9-17) were exposed to 400 Gy, POD activity was lower than WT plants (Fig. 3A). Regardless of gamma irradiation, CAT activity was higher OX-OsTPS30 plants (\#2-1, \#9-17) than WT plants (Fig. 3B). APX activity levels were increased in the OX-OsTPS30 plants (\#2-1, \#9-17) at 200 Gy gamma irradiation. However, there was no difference at 400 Gy gamma irradiation (Fig. 3C). At 0, 200 and 400 Gy gamma irradiation, SOD activity inhibition rate was $5 \sim 10 \%$ lower in the OX-OsTPS30 plants (\#2-1, \#9-17) than WT plants (Fig. 3D). These results suggested that OsTPS30 was positively regulated by regulating the activity of antioxidant enzyme involved in ROS induced by gamma irradiation.

Subcellular localization of OsTPS30

In general, monoterpene and diterpene synthases contain a transit peptides for translocation to the plastids (Tholl 2006; Arimura et al. 2009). OsTPS30 gene consists of 503 amino acid with a calculated molecular mass of $58.5 \mathrm{kDa}$. The amino acid sequence of OsTPS30 includes a DDxxD motif that has been implicated in binding with a divalent metal 
A
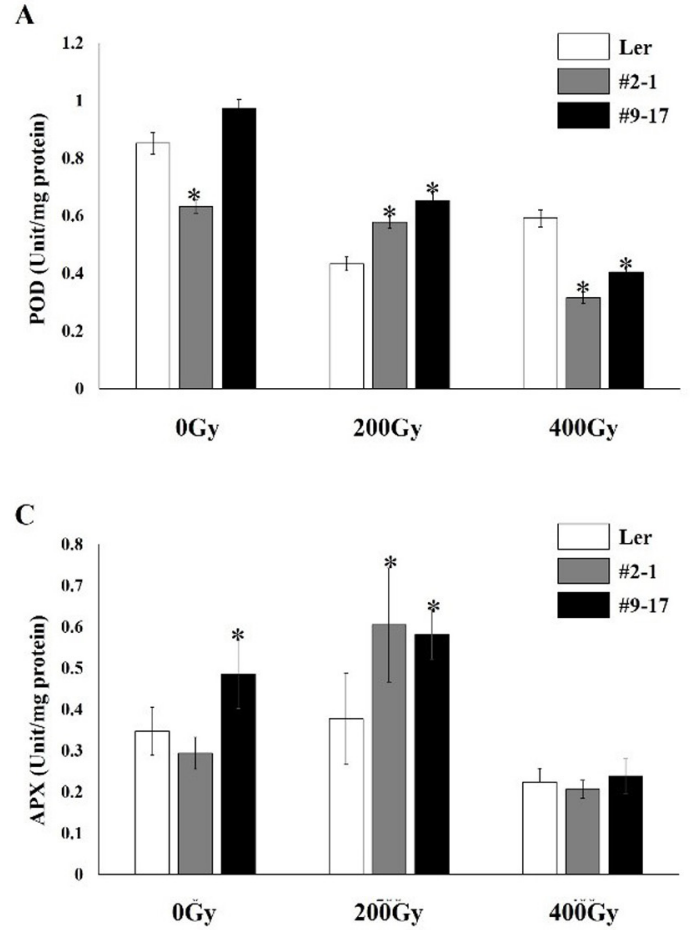

B

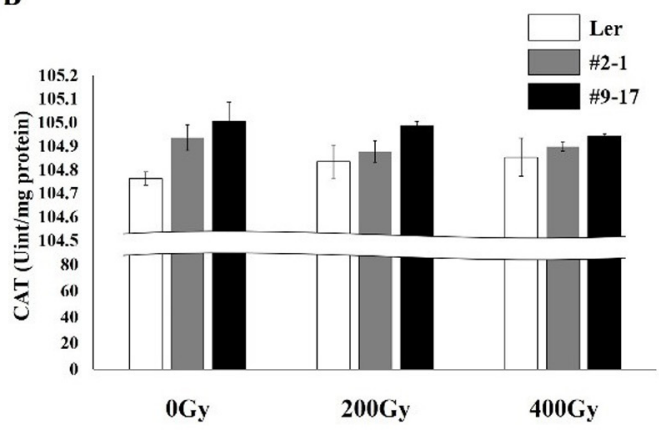

D

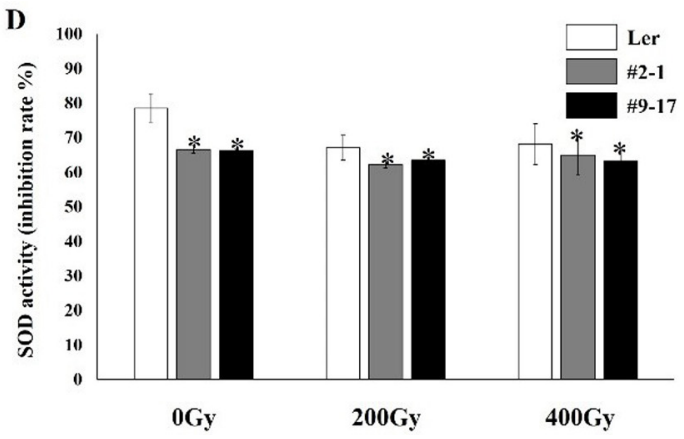

Fig. 3 Antioxidant enzyme activity of OX-OsTPS30 and WT plants after gamma irradiation. (A) POD activity. (B) CAT activity. (C) APX activity. (D) SOD activity inhibition rate (\%). Values are expressed as activity units per mg enzyme. Data is presented as the mean \pm standard deviation for the three biological replicates. Asterisks indicate significant differences in the data for the WT plants (Ler) at $P<0.05$ (One-way ANOVA)

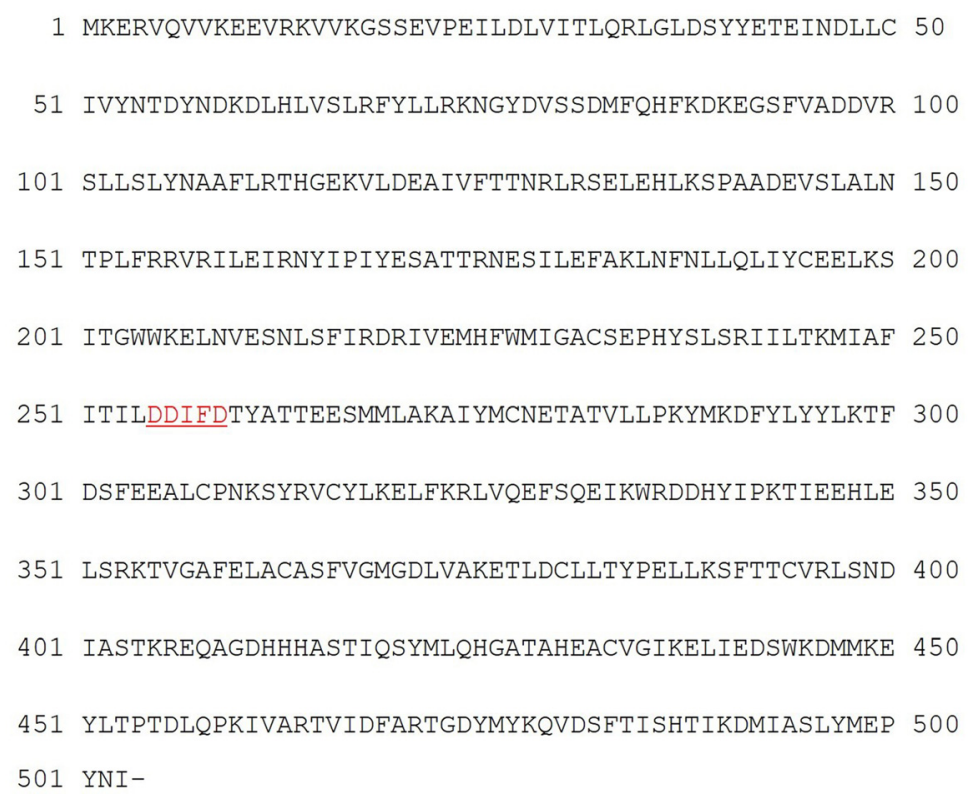

Fig. 4 Amino acid sequences of OsTPS30 (Os08g07080). The gene consists of 503 amino acids with a calculated molecular mass of $58.5 \mathrm{kDa}$. The putative conserved DDxxD motif is underline

cofactor (Fig. 4). Sequence analysis using ChloroP 1.1 (http://www.cbs.dtu.dk/services/ChloroP/) predicted the localization of OsTPS30 to the chloroplast, suggest that OsTPS30 functions as a monterpene or diterpene synthases. However, as there was no direct experimental evidence for this behavior, we isolated $A$. thaliana protoplasts from the leaves of 14-day-old WT and OX-TPS30 plants. The GFP fluorescence was monitored, which revealed that OsTPS30-GFP 


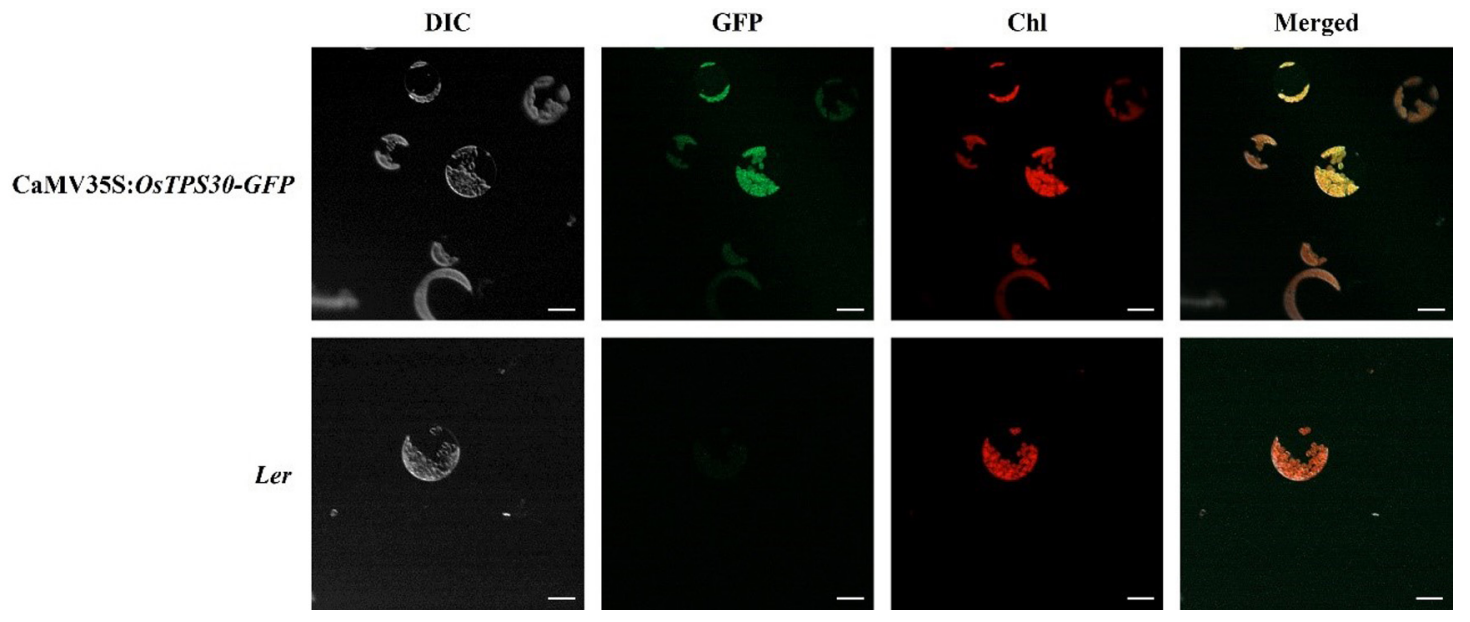

Fig. 5 Subcellular localization of the OsTPS30-GFP fusion protein in A. thaliana protoplasts. Isolated protoplasts from the leaves of OX-OsTPS30 and WT plants (Ler). Isolated protoplasts were analyzed using confocal microscopy and photographed. DIC, Differential interference contrast image; GFP, Green fluorescence protein; Chl, Red chlorophyll autofluorescence utilized as a chloroplast marker. The white bar represents $10 \mu \mathrm{m}$

fusion protein was localized in chloroplasts, suggest that OsTPS30 is a putative MEP pathway related terpene synthase (Fig. 5).

\section{Discussion}

Terpenes are defense compounds present in diverse plant species. Terpenoids play a large role in plant development and stress responses. Terpene production may be induced by abiotic stresses, including gamma-rays, UV-rays, and high temperature, ultimately generating ROS (Jenkins 2009; Esnault et al. 2010). Several aspects regarding the genetic basis of plant-responses to ionizing radiation remain unknown. Lee et al. (2015) reported that the expression levels of five OsTPS genes (i.e., OsTPS19, OsTPS20, OsTPS25, OsTPS26, and OsTPS30) are induced by gamma irradiation. However, OSTPS30 was not functionally characterized. In the current study, we observed that OSTPS30 transcript abundance increased following exposures to gamma irradiation (Fig. 1A). These results imply that OsTPS30, encodes a putative rice TPS and gamma irradiation-inducible genes.

To characterize the physiological function of OsTPS30 following gamma irradiation, we generated Ox-OSTPS30 plants. Ghorai et al. (2012) reported, linalool, monoterpene, can indirectly measure total terpenoid. As result, total terpenoid were slightly increased in Ox-OsTPS30 plants (\#2-1, \# 9-17) than WT plants (Fig. 1E). We hypothesize that the increase in the total terpenoid of Ox-OSTPS30 plants may be a reason for resistance to gamma irradiation.

Plants height and weight are a major parameter influ- encing responses to gamma irradiation. The height and weight of OX-OsTPS30 plants higher than the WT plants after the gamma irradiation (Fig. 2). Previous studies concluded that OSTPS30 expression is upregulated by gamma irradiation (Fig. 1A). Moreover, we observed that OX-OsTPS30 plants were more resistant to the gamma irradiation than the WT plants (Fig. 2). There results, the increased expression of OsTPS30 in response to gamma irradiation and greater tolerance of plants that overexpressed OsTPS30, suggest that OsTPS30 plays an important role in resistant to gamma irradiation.

Produced ROS induce damages of protein, membrane and nucleic acids and negatively influence plant growth and development. The antioxidant role of monoterpenes under oxidative stress have been experimentally elucidated to directly mitigate the ozone level leading to decreased oxidative damage (Fares et al. 2008). The POD, CAT, APX, SOD activity showed OX-OsTPS30 plants (\#2-1, \#9-17) were significantly higher than the WT plants (Fig. 3). Thus, OsTPS30 increased the activity of antioxidant enzymes, thereby eliminating ROS increased by gamma irradiation.

Terpene synthases are involved in the MEP pathway in chloroplast. Monoterpene and diterpene synthases generally contain a transit peptide that enables them to translocate to plastids (Tholl 2006; Arimura et al. 2009). Amino acid sequence analysis using ChloroP 1.1, we could predict that OsTPS30 would be present in the chloroplast. However, chloroplast transit peptide was not found. We determined that OsTPS30-GFP fusion protein is mainly localized to the chloroplast (Fig. 5), implying this OsTPS30 is a monoterpene or diterpene synthase associated with MEP pathway. In conclusion, transcription of OsTPS30 was strongly 
upregulated in rice plants after gamma irradiation. OX-OsTPS30 plants synthesized more total terpenoid than WT plants. OX-OsTPS30 plants tended to be more tolerant to gamma irradiation than WT plants. Following gamma irradiation, these OX-OsTPS30 plants showed significant differences in plant height and weight when compared to WT plants. The OsTPS30 increased the activity of antioxidant enzymes. Subcellular localization analysis of OsTPS30-GFP showed abundant fluorescent signals accumulated in the chloroplast, suggesting that OsTPS30 is putative MEP pathway-related terpene synthase.

\section{Acknowlegements}

This research was supported by grants from the Nuclear R\&D Program by the Ministry of Science and ICT (MSIT), and the research program of KAERI, Republic of Korea.

\section{Author's Contributions}

S. W. Kim designed the experimental plan and wrote the manuscript. S. W. Kim and I. J. Jung performed the experiments. J. -B. Kim, S. -Y. Kang, S. H. Kim and H. -I. Choi supervised the project. All of the authors were involved in data analysis and interpretation.

\section{References}

Aharoni A, Giri AP, Deuerlein S, Griepink F, de-Kogel WJ, Verstappen FWA, Verhoeven HA, Jongsma MA, Schwab W, Bouwmeester HJ (2003) Terpenoid metabolism in wild type and transgenic Arabidopsis plants. Plant Cell 15:2866-2884

Arimura G, Matsui K, Takabayashi J (2009) Chemical and molecular ecology of herbivore-induced plant volatiles: proximate factors and their ultimate functions. Plant Cell Physiol 50:911-923

Baldwin IT, Halitschke R, Paschold A, Von Dahl CC, Preston CA (2006) Volatile signaling in plant-plant interactions: "Talking trees" in the genomics era. Science 311:812-815

Bohlmann J, Martin D, Oldham NJ, Gershenzon J (2000) Terpenoid secondary metabolism in Arabidopsis thaliana: cDNA cloning, characterization, and functional expression of a myrcene/ (E)-beta-ocimene synthase. Arch Biochem Biophys 375:261-269

Bohlmann J, Meyer-Gauen G, Croteau R (1998) Plant terpenoid synthases: Molecular biology and phylogenetic analysis. Proc Natl Acad Sci USA 95:4126-4133

Bradford MM (1976), A rapid and sensitive method for the quantitation of microgram quantities of protein utilizing the principle of protein-dye binding. Anal Biochem 72:248-254
Chen F, Ro DK, Petri J, Gershenzon J, Bohlmann J, Pichersky E, Tholl D (2004) Characterization of a root-specific Arabidopsis terpene synthase responsible for the formation of the volatile monoterpene 1,8-cineole. Plant Physiol 135:1956-1966

Chen F, Tholl D, Bohlmann J, Pichersky E (2011) The family of terpene synthases in plants: a mid-size family of genes for specialized metabolism that is highly diversified throughout the kingdom. Plant J 66:212-229

Chen F, Tholl D, D'Auria JC, Farooq A, Pichersky E, Gershenzon J (2003) Biosynthesis and emission of terpenoid volatiles from Arabidopsis flowers. Plant Cell 15:481-494

Chen X, Chen H, Yuan JS, Köllner TG, Chen Y, Guo Y, Zhuang X, Chen X, Zhang YJ, Fu J, Nebenführ A, Guo Z, Chen F (2018) The rice terpene synthase gene OSTPS19 functions as an (S)-limonene synthase in planta, and its overexpression leads to enhanced resistance to the blast fungus Magnaporthe oryzae. Plant Biotechnol J 16:1778-1787

Cheng AX, Lou YG, Mao YB, Lu S, Wang LJ, Chen XY (2007) Plant terpenoids: Biosynthesis and ecological functions. J Integr Plant Biol 49:179-186

Clough SJ, Bent AF (1998) Floral dip: a simplified method for Agrobacterium-mediated transformation of Arabidopsis thaliana. Plant J 16:735-743

Copolovici LO, Filella I, Llusia J, Niinemets U, Penuelas J (2005) The capacity for thermal protection of photosynthetic electron transport varies for different monoterpenes in Quercus ilex. Plant Physiol 139:485-496

Dudareva N, Negre F, Nagegowda DA, Orlova I (2006) Plant volatiles: recent advances and future perspectives. Crit Rev Plant Sci 25:417-440

Esnault MA, Legue F, Chenal C (2010) Ionizing radiation: advances in plant response. Environ Exp Bot 68:231-237

Falara V, Akhtar TA, Nguyen TT, Spyropoulou EA, Bleeker PM, Schauvinhold I, Matsuba Y, Bonini ME, Schilmiller AL, Last RL, Schuurink RC, Pichersky E (2011) The Tomato Terpene Synthase Gene Family. Plant Physiol 157:770-789

Fares S, Loreto F, Kleist E, Wildt J (2008) Stomatal uptake and stomatal deposition of ozone in isoprene and monoterpene emitting plant. Plant Biol (Stuttg) 10:44-54

Fäldt J, Arimura G, Gershenzon J, Takabayashi J, Bohlmann J (2003) Functional identification of AtTPS03 as (E)-beta-ocimene synthase: a monoterpene synthase catalyzing jasmonate- and wound-induced volatile formation in Arabidopsis thaliana. Planta 216:745-751

Ghorai N, Chakraborty S, Gucchait S, Saha SK, Biswas S (2012) Estimation of total terpenoids concentration in plant tissues using a monoterpene, Linalool as standard reagent. Protocol Exchange Doi:10.1038/protex.2012.055

Herde M, Gärtner K, Köllner TG, Fode B, Boland W, Gershenzon J, Gatz C, Tholl D (2008) Identification and regulation of TPS04/GES, an Arabidopsis geranyl linalool synthase catalyzing the first step in the formation of the insect-induced volatile C16-homoterpene TMTT. Plant Cell 20:1152-1168

Irmisch S, Jiang Y, Chen F, Gershenzon J, Köllner TG (2014) Terpene synthases and their contribution to herbivore-induced 
volatile emission in western balsam poplar (Populus trichocarpa). BMS Plant Biol 14:270

Jenkins GI (2009) Signal transduction in responses to UV-B radiation. Annu Rev Plant Biol 60:407-431

Keeling CI, Bohlmann J (2006) Genes, enzymes and chemicals of terpenoid diversity in the constitutive and induced defence of conifers against insects and pathogens. New Phytol 170: 657-675

Kiryu M, Hamanaka M, Yoshitomi K, Mochizuki S, Akimitsu K, Gomi K (2018) Rice terpene synthase 18 (OsTPS18) encodes a sesquiterpene synthase that produces an antibacterial (E)-nerolidol against a bacterial pathogen of rice. J Gen Plant Pathol 84:221-229

Lee GW, Lee S, Chung MS, Jeong YS, Chung BY (2015) Rice terpene synthase 20 (OsTPS20) plays an important role in producing terpene volatiles in response to abiotic stresses. Protoplasma 252:997-1007

Lesburg CA, Zhai G, Cane DE, Christianson DW (1997) Crystal structure of pentalenene synthase: mechanistic insights on terpenoid cyclization reactions in biology. Science 277 : 1820-1824

Li G, Kollner GT, Yin Y, Jiang Y, Chen H, Xu Y, Gershenzon J, Pichersky E, Chen F (2012) Non-seed plant Selaginella moellendorfii has both seed plant and microbial types of terpene synthases. Proc Natl Acad Sci USA. 109:14711-14715

Loreto F, Förster A, Dürr M, Csiky O, Seufert G (1998) On the monoterpene emission under heat stress and on the increased thermotolerance of leaves of Quercus ilex L. fumigated with selected monoterpenes. Plant Cell Environ 21:101-107

Martin D, Fäldt J, Bohlmann J (2004) Functional characterization of nine Norway spruce terpene synthase genes and evolution of gymnosperm terpene synthases of the TPS-d sub-family. Plant Physiol 135:1908-1927

Mittler R, Zilinskas BA (1993) Detection of ascorbate peroxidase activity in native gels by inhibition of ascorbate dependent reduction of nitroblue tetrazolium. Anal Biochem 112:540-546

Moussa HR (2008) Gamma irradiation effects on antioxidant enzymes and G6PDH activities in Vicia Faba plant. J New Seeds 9:89-99

Park S, Moon JC, Park YC, Kim JH, Kim DS, Jang CS (2014)
Molecular dissection of the response of a rice leucine-rich repeat receptor-like kinase (LRR-RLK) gene to abiotic stresses. J Plant Physiol 171:1645-1653

Peters RJ, Carter OA, Zhang Y, Matthews BW, Croteau RB (2003) Bifunctional abietadiene synthase: mutual structural dependence of the active sites for protonation-initiated and ionizationinitiated cyclizations. Biochemistry 42:2700-2707

Pütter J (1974) Peroxidase. In: H.U. Bergmeyer, Ed., Methods of enzymatic analysis. Verlag Chemie Weinhan 685-690

Singh B, Sharma RA (2015) Plant terpenes: defense responses, phylogenetic analysis, regulation and clinical applications. 3 Biotech 5:129-151

Sun TP, Kamiya Y (1994) The Arabidopsis GA1 locus encodes the cyclase ent-kaurene synthetase A of gibberellin biosynthesis. Plant Cell 6:1509-1518

Sun Y, Huang X, Ning Y, Jing W, Bruce TJ, Qi F, Xu Q, Wu K, Zhang Y, Guo Y (2017) TPS46, Rice terpene synthase conferring natural resistance to bird cherry-oat aphid, Rhopalosiphum padi (Linnaeus). Front Plant Sci 8:110

Tholl D (2006) Terpene synthases and the regulation, diversity and biological roles of terpene metabolism. Curr Opin Plant Biol 9:297-304

Tholl D, Chen F, Petri J, Gershenzon J, Pichersky E (2005) Two sesquiterpene synthases are responsible for the complex mixture of sesquiterpenes emitted from Arabidopsis flowers. Plant J 42:757-771

Tholl D, Lee S (2011) Terpene specialized metabolism in Arabidopsis thaliana. Arabidopsis Book 9:e0143

Yamaguchi S, Sun TP, Kawaide H, Kamiya Y (1998) The GA2 locus of Arabidopsis thaliana encodes ent-kaurene synthase of gibberellin biosynthesis. Plant Physiol 116:1271-1278

Yoshitomi K, Taniguchi S, Tanaka K, Uji Y, Akimitsu K, Gomi K (2016) Rice terpene synthase 24 (OsTPS24) encodes a jasmonate-responsive monoterpene synthase that produces an antibacterial $\gamma$-terpinene against rice pathogen. J Plant Physiol 191:120-126

Zhang P, Fuentes S, Siebert T, Krstic M, Herderich M, Barlowa ER, Howell K (2016) Comparison data of common and abundant terpenes at different grape development stages in Shiraz wine grapes. Data Brief 8:1127-1136 